\title{
Students' understanding of non-inertial frames of reference
}

\author{
S. Küchemann, ${ }^{1, *}$ P. Klein, ${ }^{1}$ H. Fouckhardt, ${ }^{2}$ S. Gröber, ${ }^{1}$ and J. Kuhn ${ }^{1}$ \\ ${ }^{1}$ Department of Physics, Physics Education Research Group, Technische Universität Kaiserslautern, \\ Erwin-Schrödinger-Strasse 46, 67663 Kaiserslautern, Germany \\ ${ }^{2}$ Department of Physics, Integrated Optoelectronics and Microoptics Research Group, Technische \\ Universität Kaiserslautern, Erwin-Schrödinger-Strasse 46, 67663 Kaiserslautern, Germany
}

(Received 26 February 2019; accepted 4 March 2020; published 24 March 2020)

\begin{abstract}
The concepts of the Coriolis and the centrifugal force are essential in various scientific fields and they are standard components of introductory physics lectures. In this paper, we explore how students understand and apply concepts of rotating frames of reference in the context of an example lecture demonstration experiment. We found in a predict-observe-explain setting that after predicting the outcome prior to the demonstration, only one out of five physics students correctly reported the observation of the trajectory of a sphere rolling over a rotating disc. Despite this low score, a detailed analysis of distractors revealed a significant improvement in the distractor choices during the observation of the experiment. In this context, we identified three main preconceptions and learning difficulties: The centrifugal force seems to be only required to describe the trajectory if the object is coupled to the rotating system, the inertial forces cause a reaction of an object on which they act, and students systematically mix up the trajectories in the stationary and the rotating frame of reference. Furthermore, we captured students' eye movements during the predict task and found that physics students with low confidence ratings focused longer on relevant task areas than confident students despite having a comparable score. Consequently, this metric is a helpful tool for the identification of preconceptions using eye tracking. Overall, the results help us to understand the complexity of concept learning from demonstration experiments and provide important implications for instructional design of introductions to rotating frames of reference.
\end{abstract}

DOI: 10.1103/PhysRevPhysEducRes.16.010112

\section{INTRODUCTION}

Rotating frames of reference play an important role in a variety of fields in physics. Accordingly, Coriolis and centrifugal terms need to be considered for an accurate account of the theoretical description of the motion of an object in rotating reference frames. While the Coriolis force was originally introduced to describe the energy transfer in water wheels, nowadays it is applied to problems in meteorology [1,2], oceanography [3], astrophysics [4], optics [5], and nuclear physics [6]. Given this wide range of applications, the Coriolis and the inertial centrifugal force (ICF) are sometimes topics in introductory physics courses in college-level education and, accordingly, there are a large number of experiments and open online materials [7-13] which intend to demonstrate the Coriolis effect, i.e., the apparent deflection of an object by the Coriolis force. Moreover, there are several mechanics textbooks which

\footnotetext{
*s.kuechemann@physik.uni-kl.de
}

Published by the American Physical Society under the terms of the Creative Commons Attribution 4.0 International license. Further distribution of this work must maintain attribution to the author(s) and the published article's title, journal citation, and DOI. derive the equation of motion of objects in non-inertial frames of reference [14-22] or contain a descriptive approach to the topic [23-27]. However, there are several shortcomings and false accounts outlined below, potentially causing incoherent conceptions and complications in students' understanding.

In this paper, we explore how students understand and apply concepts of rotating frames of reference in the direct context of an example lecture demonstration experiment. Therefore, we identify and study relevant preconceptions, the non-obvious learning effect of experiment observation, and the relationship between response security and duration of focus on relevant areas (as measured by eye tracking).

The paper is structured in the following way. After this introduction, an overview of the current state of research and the preliminary work follows in the second section and the third section explains the materials and methods used in this work. The subsequent section contains the results of the predict-observe-explain (POE) test including self-confidence ratings, student interviews, and eye-tracking data in the context of an example demonstration experiment of rotating frames of reference. Then, these results are discussed in the context of previous literature and, eventually in the final section, we conclude the manuscript with the main consequences of the results for physics education research. 
In this work, we address three research questions:

(1) What is the influence of a lecture experiment demonstrating the motion of objects in non-inertial frames of reference on learning about the outcome of the demonstration?

(2) What are the prevailing preconceptions of physics students in the field of non-inertial frames of reference?

(3) Is there a specific eye-movement pattern that relates to the performance or confidence of physics students within a POE setting?

\section{CURRENT STATE OF RESEARCH AND PRELIMINARY WORK}

\section{A. Simplified conceptions of the Coriolis effect and the centrifugal force}

A theoretical framework containing the equation of motion of an object described in a rotating frame of reference (RFR) (also termed non-inertial frame of reference) is provided in Sec. A 1. In simplified depictions of a curved trajectory of an object in a RFR, the Coriolis force is often presented as the only cause for the deflection [7-13]. However, according to Eq. (A2) (see Appendix), the ICF also acts on the object in a vector sum with the Coriolis force. The fact that the ICF is a necessary quantity to describe the trajectory of an object in a rotating frame of reference can be understood from two arguments of a thought experiment.

(I) Let us imagine a situation where a plane flies in a uniform motion over a large rotating disc starting from the center of rotation. If an observer located on the disc used the Coriolis force for the description of the curved trajectory only, they would calculate that the plane returns to the center of rotation at some point in time because the Coriolis force is always perpendicular to the direction of motion thus leading to a circular trajectory. For an observer in an stationary frame of reference (SFR), however, it is obvious that this case would not occur because the plane flies in a uniform motion due to the absence of any real force. In reality, the plane would pursue a spiral trajectory for the observer in the RFR which is the consequence of the vector sum of the inertial centrifugal force and Coriolis force.

(II) During the aforementioned motion of the plane, the absolute value of the velocity $\left|\vec{v}^{\prime}\right|$ in the RFR would increase according to Eq. (A1) because $\vec{v}^{\prime}$ is a vector sum of two perpendicular vectors, the velocity in the SFR $\vec{v}$, which is constant and the inverse transverse velocity $-\vec{\omega} \times \vec{r}$ of the rotating disc which increases with increasing distance $r$ to the center of rotation (see Sec. A 2 for a derivation). Since the Coriolis force is always perpendicular to the direction of motion (given by $\vec{v}^{\prime}$ ), it cannot be the reason for this apparent increase in $\left|\vec{v}^{\prime}\right|$. Only the centrifugal force that points outwards from the center of rotation can be responsible for this effect.

\section{B. Why does the concept of inertial forces potentially cause problems for students?}

In some depictions of RFR the centrifugal force is neglected when an object, which is moving in a RFR, does not experience an interaction to the RFR. For instance, in Ref. [28], the author states that "the centrifugal effect is eliminated if there is no interaction between the rotating disk and the body, like a ball rolling on the disk without friction." This preconception that the centrifugal force only occurs, when the object is somehow coupled (e.g., by friction or a rope) to the rotating system [28] is potentially guided by empirical experiences, such as the feeling of a force pointing outwards when sitting in a carousel or in a car driving through a turn, i.e., the body reacts to the force because the person is actually partially coupled to the rotating system. This seems to be in conflict with the characteristic that fictitious forces do not occur as a actionreaction pair with another force [29]. This cognitive dissonance can be resolved by discriminating between the centrifugal force which occurs as a consequence to a centripetal force (here termed "reactive centrifugal force," RCF, see Appendix, Sec. A 3 for an example of the RCF) and the one which occurs as a fictitious force in a RFR ("inertial centrifugal force," ICF) [30] which occurs as a consequence of a description from a rotating frame of reference. Sometimes textbooks and scientific articles lack this helpful linguistic distinction between RCF and ICF $[14,17,28,31,32]$. The reason for this could be that the mathematical equations are the same, only the situations in which they occur and how they are perceived are different. For the occurrence of the RCF, a coupling to the RFR is indeed required so it occurs when a centripetal force occurs. For the occurrence of the ICF, a coupling to the RFR is not required. Accordingly, the occurrence of a centripetal force can be perceived (and might be related to the occurrence of the RCF) when driving through a turn or sitting in a merry go round, whereas the occurrence of the ICF, for instance, for a passenger in an airplane flying in a uniform motion over a rotating disc, cannot be felt.

Apart from the RCF and ICF, a centrifugal force term may also occur as a d'Alembert's force as pointed out by Kobayashi, which is an inertial resistance and occurs as a consequence of a coordinate transformation [30] to plane polar coordinates and has the form "mass $\times$ centripetal acceleration." The centrifugal force as an inertial resistance is not part of the work here but certainly adds to the complexity in teaching and learning of inertial forces.

\section{Experimental lecture demonstrations and students' understanding of the Coriolis force}

Lecture demonstrations in the classical sense mean the demonstrations of experiments by the lecturer during class 
while the students passively observe the presentation. The intention of the lecturer is often that the students process the information and understand their observations by integrating it into their conceptual knowledge [33]. Unfortunately, despite their regular use in introductory physics lectures, it has been shown that demonstrations will have little effect on students' conceptual learning if the students passively observe the experiments [34]. At the same time, the correct observation of a lecture demonstration is a necessary prerequisite for conceptual learning [33]. Please note that the phrase "correct observation" refers to the ability to correctly report the outcome of a lecture demonstration, such as a trajectory of an object or a velocity change, without applying physical principles or relating it with previous knowledge.

In this context, POE is an interactive teaching scenario that can be implemented during experimental lecture demonstrations [35-37]. While it is sometimes proposed as an eight-step approach, here we reduce it to three central steps $[38,39]$. First, in the predict phase, the students are asked to make an educated guess of the outcome of the experiment. This step helps to initiate learning processes by reflecting on and relating to theoretical backgrounds and thus forming a mental model which links the theory to the experiment [39]. In the second, i.e., the observe phase, the experiment is demonstrated and the students visually perceive its process and outcome. Here, students are expected to relate their observation to the previously anticipated result and, consequently, approve or reconsider their mental model [36]. In the final, i.e., the explain phase, the outcome of the experiment is revisited, typically by the teacher. In this part the teacher explains the established link between the theory and the outcome of the experiment. In this way, using POE in class provides the opportunity for the teacher to interact with the students and it can be used to stimulate discussions among peers.

In our work, we included the predict phase because we intended to assess to pre-instructional and predemonstrational student understanding about the experiment. These data were important since the students were already previously exposed to the topic (see below) and the combination of the datasets from the predict and the observe phase allowed us to conclude on the learning gain during the demonstration. Additionally, we intended that the students ideally retrieve conceptual knowledge prior to the demonstration.

When a student receives an instruction or is confronted with a task about a certain topic, he or she typically has already a certain conception about the situation [40]. These preinstructional conceptions of students on the content have been studied and documented in various science contexts since several years [41]. The identification of such student conceptions plays an important role for instructional design and it is addressed in the context of conceptual change, which refers to a pathway towards the acquisition of conceptual understanding [42]. To our knowledge there has not been any quantitative study which examines students' conceptions of Coriolis force and inertial centrifugal force so far. Stommel et al. report that students consider the Coriolis effect as "mysterious" phenomenon and a result of "formal mathematical manipulations" as pointed out by Persson $[28,32]$. Previously observed conceptions of students in mechanics imply, for instance, the "motion implies a force" conception $[43,44]$. This conception potentially still persists in the students' understanding, thus complicating the students' conceptual learning of rotating frames of references and may translate to our study. We have accounted for these potential student difficulties in the post-test. In our study we do not discriminate whether or not a student's preconception is based on a coherent construct or is a collection of unstructured fragments [40].

\section{Analysis methods}

\section{Eye tracking in educational research}

During the POE tasks and the instruction (between observe and explain) we have recorded the students' eye movements. In the analysis of this eye-tracking data, researchers usually discriminate between fixations and saccades. While fixations are relatively long periods in which the eye does not move, saccades are very fast movements of the eye between fixations. In other words, a saccade is considered as the motion between two (or more) consecutive fixations, typically lasting less than $100 \mathrm{~ms}$. Based on the eye-mind hypothesis [45], fixations reflect the focus of attention and indicate information processing, whereas information intake is suppressed during saccades.

In general, the eye-tracking technique has gained growing attention in educational research in the past years, since several cognition-psychological and educational questions can be addressed with this method. It has been proven to be a powerful tool to complement qualitative and quantitative research with a data resource on the behavioral level [46]. Analyzing the visual attention of students has the potential for identifying students' conceptions and learning difficulties [47-50]. Thus, eye tracking can provide a promising base to design effective instructions to help students learning, e.g., by the integration of visual cues [51,52], it can reveal student strategies during problem solving [53], and it is able to discriminate between expert and novice eye-gaze patterns [54].

In this context, Gegenfurtner et al. concluded in a metaanalysis that experts, in comparison to non-experts, have shorter fixation duration, but more fixations on relevant areas and longer saccades [55], confirming a number of theories, such as the theory of long-term working memory [56] and the information-reduction hypothesis [57].

In the area of physics education research, eye tracking has recently received attention for quite different purposes. Some studies used eye tracking to compare the visual 
attention during problem solving, e.g., while students were taking a multiple choice test [50,58-62]. In this vein of research, Ohno et al. investigated time and location measures on the Force Concept Inventory and found differences between students with and without correct Newtonian views [58]. Students spent more time looking at areas that contained relevant information to solve the problem. Similarly, Kekule found that better performing students focused more on important features when answering questions about kinematics graphs [59,60]. Rosengrant et al. analyzed the transitions between the problem statement involving an electrical circuit and the answer choices. They found that expert students more frequently looked back at the problem diagram than novice students did [61]. Applying deeper analysis procedures on saccadic data in the context of vector field plots, Klein et al. found that expert students differed from novice students by the ability to suppress irrelevant saccades which, in this case, refer to those saccades that do not follow the direction of the axes [62]. The same analysis procedure revealed that physics students follow the curve in a line graph with their eyes more often than nonphysics students when answering questions about graph slope, indicating correct cognitive processing of the slope concept [50].

Other studies used eye tracking to analyze learning processes of students, e.g., with multimedia, and compared the effects of interventions on students' eye movements and performance [51,52]. Madsen et al. exposed students randomly to two conditions which differed by whether the participants saw conceptual physics problems overlaid with dynamic visual cues or not [51]. Students in the cue condition were shown an initial problem, and if they answered incorrectly, they would have been shown a series of problems each with selection and integration cues overlaid on the problem diagrams. Students in the non-cue condition were also provided a series of problems, but without any visual cues. The authors found that significantly more participants in the cue condition answered the problems super-imposed with visual cues correctly and spent significantly less time looking at irrelevant areas of the diagram. Thus, the use of visual cues to influence visual attention and to foster problem solving was shown to be effective. Additionally, Madsen et al. found that the response accuracy is correlated to focus duration on relevant areas [47].

To support the connection across two types of complex representations, Klein et al. designed two instructions and tested their impact on students performance [52]. The instructions included written explanations, equations, and visual representations, but differed in the presence of visual cues. The results show that students instructed with visual cues performed better, responded with higher confidence, experienced less mental effort, and rated the instructional quality better than students instructed without cues. Furthermore, it was shown that cognitive integration processes appear in both groups at the same point in time, but they were significantly more pronounced for students in the cue condition, reflecting a greater attempt to construct a coherent mental representation during the learning process.

Apart from that there are several studies that are peripherally related to physics education research, such as trouble shooting of malfunctioning circuits [63], investigations of students understanding about how mechanical systems work [64], and how spatial ability is related to eye movement data when predicting trajectories [65]. To our knowledge, eye tracking has not been applied in the context of demonstration experiments in POE settings so far.

\section{Self-confidence ratings}

In this study, we use self-confidence ratings after the students have answered a question. These metacognitive ratings in a single choice format reflect the ability of students to self-monitor their thought processes, which comprises a reflection of the understanding of the topic and the performance in the task $[66,67]$. In common interpretations of confidence ratings, the difference between the confidence rating and the accuracy of the response is termed bias. The bias is low for any student who has a comparable confidence rating to his or her accuracy and, consequently, it would be high if the student tends to overestimate or underestimate his or her performance. The level of the bias is an indication for the calibration, which describes the relationship between response correctness as a function of confidence ratings [68]. The relatively robust effect of overconfidence can be explained within the probabilistic mental model (PMM) theory proposed by Gigerenzer et al. [69,70], in which confidence judgments are first a spontaneous consequence of a local mental model (LMM). In cases where a LMM in the context of a specific task fails, a PMM is created, in which the person retrieves probabilistic cues from the environment. The mismatch between the cue validity and ecological validity, where the latter one refers to the true account of a certain situation, might be one of two reasons for an overconfidence. The second potential reason within the PMM theory is that the set of information retrieved from the environment is not a representative selection for the reference set [69] and, for comparison, the reason for an overconfidence is not an incorrect perception of the task difficulty [71,72].

In the field of physics education, Planinic et al. found significantly higher confidence ratings for wrong answers in the area of Newtonian dynamics than in the area of electrical circuits, suggesting that concepts of Newtonian dynamics are more prone to incoherent conceptions [73].

In this work, we use the confidence ratings as an aid to identify underlying conceptions which reveal themselves when the student appears to be rather confident with an incorrect answer. Furthermore, this study explores the influence of the level of calibration on the conceptual learning within a POE setting and relates the confidence to eye-tracking metrics. 


\section{MATERIALS AND METHODS}

\section{A. Participants}

The sample consists of 21 freshman students ( 4 female, 17 male) with a physics major at the Technische Universität Kaiserslautern, Germany. The students were participants of the lecture "Experimental Physics 1" (total number of participants in the lecture is 52,8 female and 44 male), where they had seen experimental lecture demonstrations (different to the one used in this study) and the mathematical derivation of the topic of non-inertial frames of reference in one lecture, one tutorial, one problem sheet, and one recitation session prior to participation in this study. The average final exam score of all students in the lecture Experimental Physics 1 was $54 \%$ and the average final exam score in this lecture of those students participating in this study was $61 \%$. Participation in this study was voluntary and was compensated with 10 euro. The study took place several weeks before the final exam of the lecture and the students expected that the topic might be part of the exam.

One condition for students to enroll in a study program at a German university is an entrance qualification which is typically achieved via passing the "Abitur," the final high school diploma. For this high school diploma, the standards in math and in physics are listed in Refs. [74,75], respectively. This means that before entering a study program, students had typically covered differentiation and integration of one-dimensional linear and nonlinear functions with real domain in math, and in physics, high school programs cover uniform and accelerated motion but no Galilean transformation or non-inertial frames of reference. At the university, freshman physics students usually attend at least one math course during the first semester which covers topics of multidimensional differentiation (such as gradient, divergence, rotation, and integration) complex numbers, and differential equations. In physics, the students learn about Newton's mechanics and review uniform and accelerated motion including Galilean transformation and non-inertial frames of reference.

\section{B. Experimental setup}

The setup consists of a rotating disc with a diameter of $55 \mathrm{~cm}$ that is connected to a motor that allows the disc to rotate at a constant angular velocity [see Figs. 1(a) and 1(b)]. Initially, the sphere rests at the end of a tilted rail which is attached to the rotating disc pointing in the direction of the center of rotation (since the rail is attached to the rotating disc the sphere receives an initial tangential velocity component). As soon as the rail passes a trigger, the sphere starts to roll down the rail (from this acceleration the sphere receives an initial radial velocity component). The experiment is recorded from the top via two cameras. The first camera is connected to the stationary frame and does not move while the disc rotates. The second camera is attached to the rotating disc,

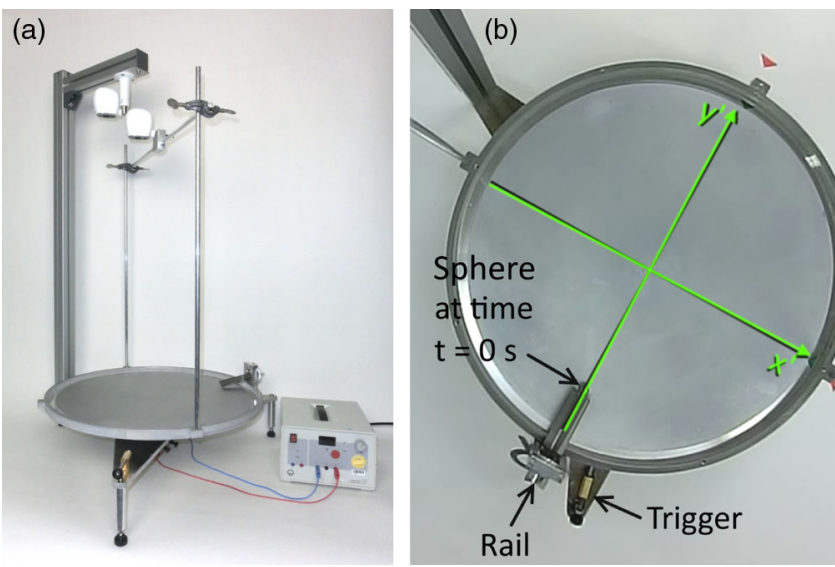

FIG. 1. (a) The experimental setup for the demonstration of rotating frames of references. (b) Top view of the rotating disc.

allowing the observation from a perspective of an observer in a non-inertial frame of reference.

In the SFR, the sphere moves uniformly in a straight line on the left side of the disc in respect to the center of rotation. Note here that it does not run through the center because the resulting motion is a superposition of the tangential and the radial part.

\section{Study design}

The study design is outlined in Fig. 2. The pretest consisted of three single choice items in a paper-pencil test assessing the understanding of essential representations of vectors. Thus, we verified whether or not the students had visual understanding of typical depictions of rotating frames of reference used in this study-a necessary prerequisite for learning from multiple visual representations as in the instruction part [76] (see Supplemental Material [77] for pretest questions). It was followed by an explanation of the experimental setup and the procedure of the experiment (without demonstration yet) by the instructor (see Fig. 2). In this phase, the students were allowed to ask questions.

Afterwards, the students were asked two questions to anticipate the trajectory of the sphere in a stationary frame of reference (first) as well as in a rotating frame of reference (second), each of them in a single choice format which represent the predict phase. These two questions were

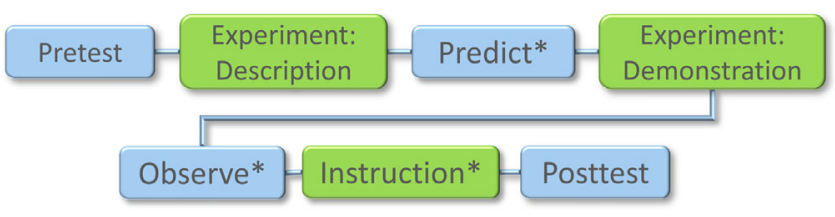

FIG. 2. Study design where $*$ indicates the parts in which the eye movements were recorded. Blue-colored boxes refer to those parts where students answered questions and green boxes are those parts in which students received information. 
computer based and the eye movements were recorded. After each prediction, the students were asked to rate their confidence on a four-point Likert scale ranging from "very confident" to "very unconfident."

Then, the instructor demonstrated the experiment twice without making any comments or giving explanations (part "Experiment: Demonstration"). The students were standing in front of the experiment and were allowed to walk around the experiment in order to observe it from their preferred perspective. This part was supposed to closely resemble an ideal situation of a lecture demonstration. Then, the students were asked to answer the same two questions as in the predict phase in order to report their observation of the trajectory in the inertial and in the non-inertial frame of reference (part observe). Again, we used eye-tracking and confidence ratings for these two computer-based items.

Subsequently, the students received the computer-based instruction consisting of two text pages and six videos. The first page displayed a standard textbook instruction of inertial forces including the equations of the Coriolis and centrifugal force. The second page explained the trajectory of the sphere rolling over a rotating disc in the particular context of the previously demonstrated experiment (see Supplemental Material [77]). In this figure the disc rotated in the same direction and all conditions (angular velocity of the disc, tilting angle of the rail, sphere) were the same as during the demonstration. This page also contained two snapshots of the final location of the sphere during the experiment from each perspective (see Fig. 3) augmented with circles and arrows indicating the trajectory and velocity vectors in both frames of reference, respectively.

After this first instruction page, three videos from each of the two perspectives (i.e., six videos in total) were shown to the students. The first video showed the experiment recorded by the stationary camera in real time. It was augmented with the same information as in the snapshots in Fig. 3. The two following videos were identical to the first
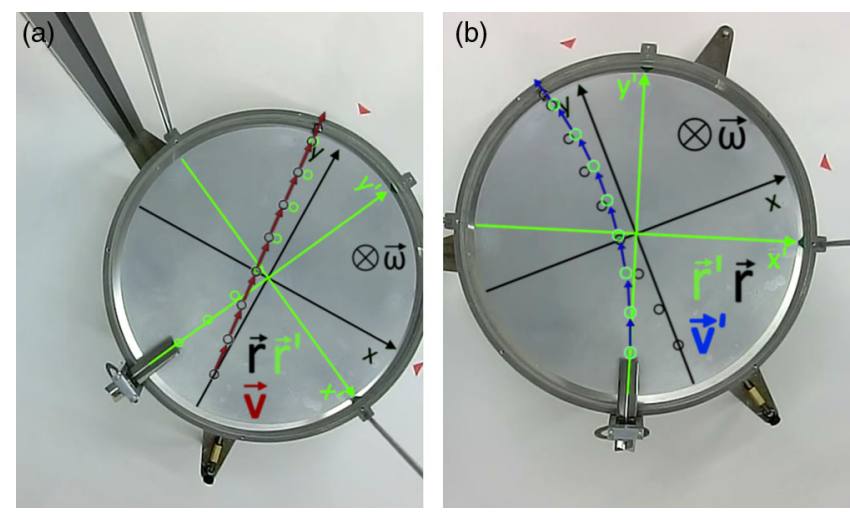

FIG. 3. Snapshots of augmented videos during the final phase of the experiment in the stationary frame of reference (a) and the rotating frame of reference (b). The black coordinate system $K$ (axes $x$ and $y$ ) is stationary and the green coordinate system $K^{\prime}$ (axes $x^{\prime}$ and $y^{\prime}$ ) rotates at the same angular velocity as the disc. one but they were played in slow motion $(4 \times$ slower). The fourth video was recorded from the rotating camera and was displayed in real time. The two following videos were identical to the fourth one but they were played in slow motion $(4 \times$ slower). The students had no option to pause or replay the videos.

After the instruction, the post-test in a paper-pencil format and two computer-based questions followed. It consisted of seven true-false items, two items explain (identical to predict and observe) and seven single choice items, two of which had a direct link to the experiment and visual attention was recorded during these two questions using eye-tracking. Here, the analysis was restricted to the POE and the interview items. After completing the posttest, the students were asked to comment on their responses of two single choice items from the post-test in an audio interview (see Supplemental Material [77] for items). The aim of the interview was to reveal students' conceptions. Therefore, these two questions were directly motivated by the misleading depictions in literature (see above). From the results of the post-test, in this work, we report the analysis of the two items explain and the two interview items.

\section{Eye-tracking equipment}

The motions of the eyes were recorded using a Tobii X3-120 stationary eye-tracking system with a visual-angle resolution of $0.40^{\circ}$ and a sampling rate of $120 \mathrm{~Hz}$. The questions were presented on a 22-inch computer screen with a resolution of $1920 \times 1080$ pixels and refresh rate of $75 \mathrm{~Hz}$. The eye-tracking system was operated and the data were analyzed using the software Tobii Studio.

\section{RESULTS}

\section{A. Test scores of POE items}

The test scores of the POE questions are shown in Fig. 4(a). The score in each POE part is the average score from two questions about the trajectory of the sphere on the rotating disc: The first question is about the trajectory in a stationary coordinate system and the second one is about the trajectory in a rotating coordinate system.

It is noticeable that the students have very low scores during the predict phase in both frames. The demonstration of the experiment, which is the only intervention between predict and observe, has no statistically significant effect on the score $(p=0.27)$ which means that $80 \%$ of the students are unable to report the trajectory of a sphere in two coordinate systems after observing the experiment twice. After the instruction including a theoretical textbook introduction, two augmented photographs (see Fig. 3) and six videos, in the part explain, only $38 \%$ of the students report the trajectories of the sphere incorrectly.

To evaluate the change in confidence ratings between predict and observe we performed two nonparametric 

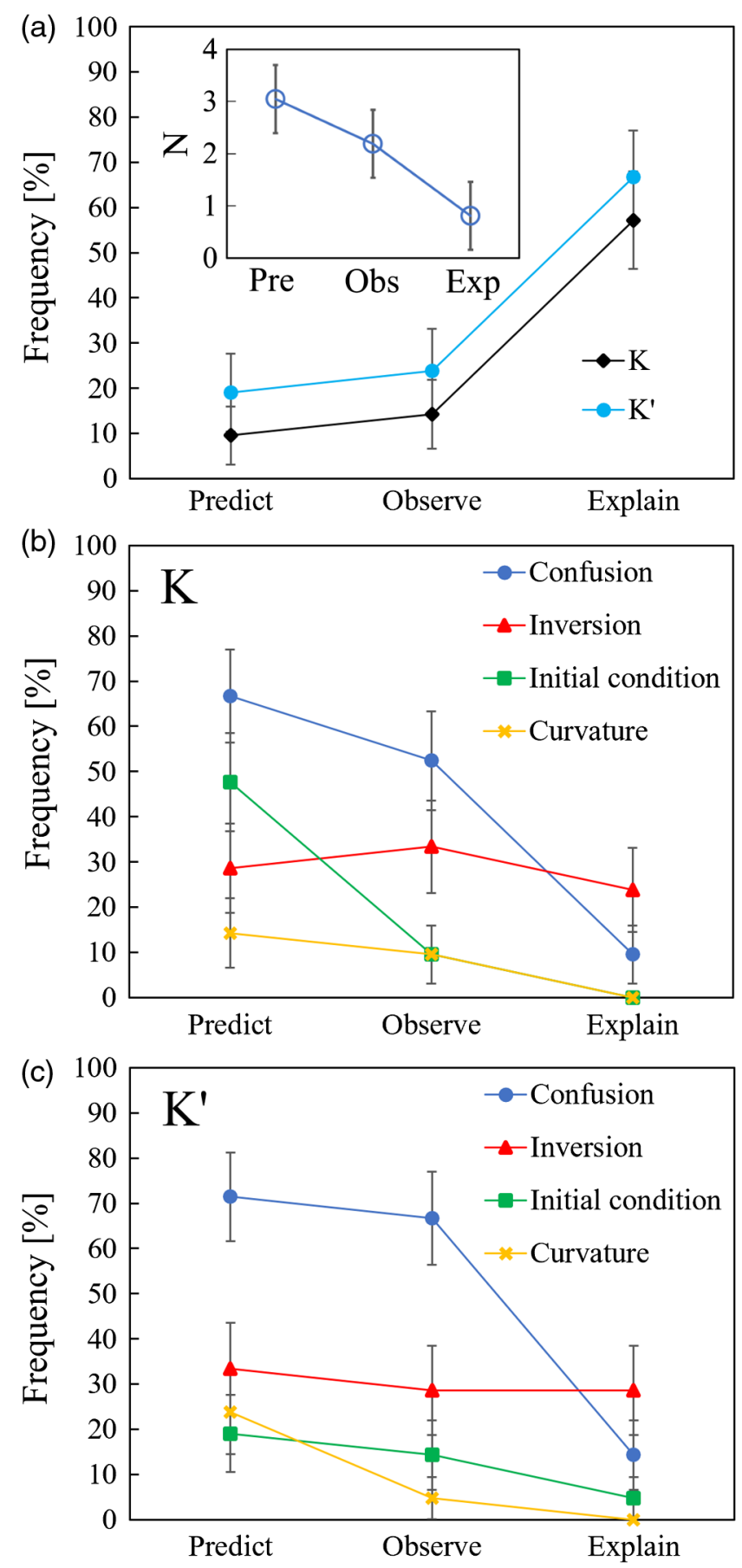

FIG. 4. (a) Test scores of POE items in the inertial frame K and the non-inertial frame $K^{\prime}$. Frequency of different distractor groups in the inertial frame (b) and in the non-inertial frame (c). The inset in panel (a) shows the average total number of distractor categories of the POE items which refers to the analysis in Sec. IV B (see also Table I).

Wilcoxon signed rank tests with continuity correction. We found that there are no statistically significant differences in the confidence ratings between predict and observe in the inertial frame of reference (average confidence ratings in $K$ : predict, $C=2.2 \pm 0.5$; observe, $C=1.9 \pm 0.9$,

where $1=$ very confident and $4=$ very unconfident, $p=0.19)$ as well as in the non-inertial frame of reference (average confidence ratings in $K^{\prime}$ : predict, $C=2.2 \pm 0.9$; observe, $C=2.2 \pm 0.8, p=0.77)$.

\section{B. Analysis of distractors}

For a deeper understanding of error sources and the influence of interventions, we assigned the distractors of the POE items to different different categories (see Figs. 4b and $4 \mathrm{c}$ for the frequencies of each category). The distractors are displayed in Fig. 5. As mentioned above, the sphere does not run through the center of rotation because the resulting motion is a superposition of the tangential and the radial part. This means that option (b) is correct in the inertial frame of reference $K$ (Fig. 5). In the non-inertial frame, the trajectory (d) describes the motion correctly (see Fig. 5). The following example may demonstrate the motivation for this categorization.

A student who chooses an option which depicts a straight trajectory through the center of rotation in the inertial frame of reference $K$, is likely to have a different perception of the trajectory and a potentially different concept of the situation than a student who chooses a curved trajectory which is deflected to the right of the center in $K$, despite the fact that both answers are incorrect. However, in this study we only have the opportunity to characterize the distractors regarding certain surface features, but cannot conclude on specific errors the students made or conceptions the students had. Despite this restriction the categorization into different
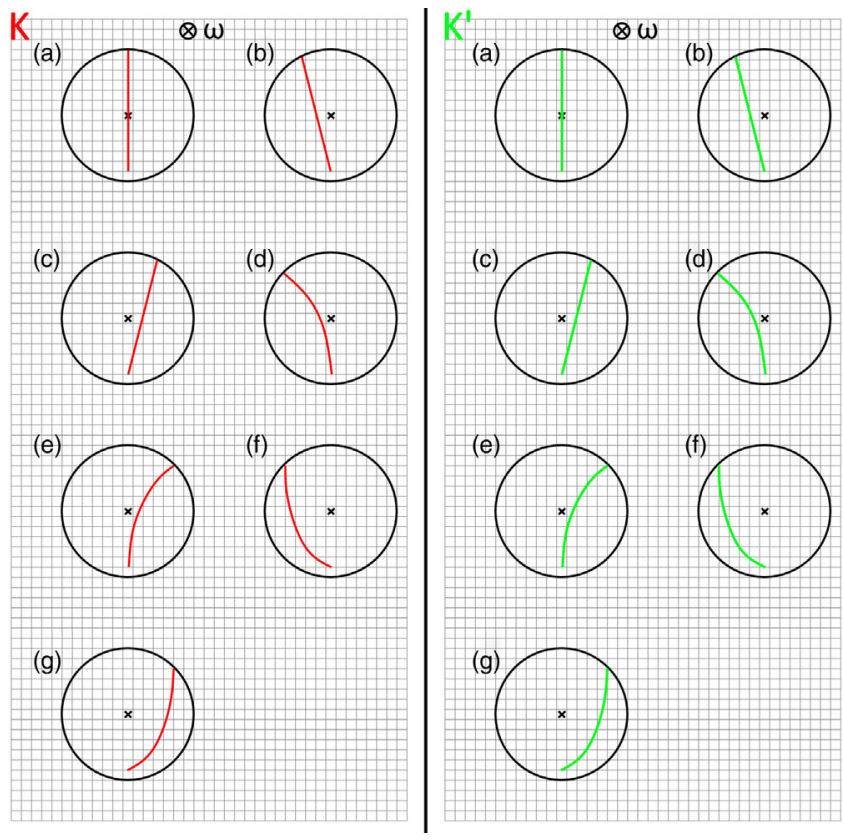

FIG. 5. Alternative answer options of the POE items in the inertial frame of reference $K$ and the non-inertial frame of reference $K^{\prime}$. In both coordinate systems the distractors are identical. 
distractor groups provides helpful insights in order to see the effect of the demonstration.

In detail, we identified four different distractor groups within the questions of the POE task:

(I) Confusion of $K$ and $K^{\prime}$ : All those distractors belong to this category which display a curved trajectory in $K$ or a straight trajectory in $K^{\prime}$. In $K$, the distractors (d), (e), (f), and (g) are included. In $K^{\prime}$ the distractors (a), (b), and (c) are included.

(II) Inversion: All distractors which depict a trajectory to the right in respect to the center of rotation belong to this group. Included distractors in $K$ : (c), (e), (g). Included distractors in $K^{\prime}$ : (c), (e), (g).

(III) Initial condition: Here, the distractor which does not consider that the sphere also has a tangential velocity component belongs to this category. Included distractors in $K$ : (a). Included distractors in $K^{\prime}$ : (a).

(IV) Curvature: This group represents all distractors which display a wrong curvature. Included distractors in $K$ : (f), (g). Included distractors in $K^{\prime}$ : (f), (g).

Following this line of thought, the assignment of the distractor groups to the different answer options of the POE items implies that some distractors belong to more than one group (see Table I). The number $N$ of distractor groups for one answer option ranges from $0-3$ in $K$ and $0-2$ in $K^{\prime}$. Figure 4(b) shows the frequency of each distractor group in the questions of the inertial frame during the POE tasks. It is noticeable that, as a consequence of the experiment demonstration, particularly the frequency of category III ("initial condition") is reduced between predict and observe in the inertial frame of reference, i.e., the students were less likely to choose a distractor which shows that the sphere rolls through the center of rotation. While the frequency of distractors in category I decreases only slightly between predict and observe, the frequency of group II ("inversion") and IV ("curvature") does not change as a result of the experiment demonstration.

Figure 4(c) shows the frequency of each distractor group in the questions of the non-inertial frame during the POE tasks. Here, as a consequence of the experiment demonstration, the frequency of category IV decreases between predict and observe, i.e., in the non-inertial frame the students were less likely to choose a distractor that shows a

TABLE I. Distractor category (DC) and number of distractor categories $(N)$ for each answer alternative in $K$ and $K^{\prime}$.

\begin{tabular}{lcccc}
\hline \hline Distractor & DC $_{K}$ & $N_{K}$ & DC $_{K^{\prime}}$ & $N_{K^{\prime}}$ \\
\hline$a$ & III & 1 & I, III & 2 \\
$b$ & cor & 0 & I & 1 \\
$c$ & II & 1 & I, II & 2 \\
$d$ & I & 1 & cor & 0 \\
$e$ & I, II & 2 & II & 1 \\
$f$ & I, IV & 2 & IV & 1 \\
$g$ & I, II, IV & 3 & II, IV & 2 \\
\hline \hline
\end{tabular}

curvature that is inconsistent with the trajectory of the sphere. Additionally, the frequencies of distractors in categories I, II, and III do not change as a result of the experiment demonstration.

Summing up the number of distractor categories of both reference frames for each participant, the average difference between the total number of distractor groups in predict $(N=3.05 \pm 1.40)$ and observe $(N=2.19 \pm 1.29)$ exhibits a significant medium effect [Cohen's $d=0.64$, $p<0.05$, see inset of Fig. 4(a)]. In comparison, the average difference between the number of distractor categories in observe and explain $(N=0.81 \pm 1.03)$ exhibits a significant, very large effect (Cohen's $d=1.18, p<0.001$ ). The largest improvement between predict and observe was found in the observation of the initial condition, i.e., the students were able to correct their prediction that the sphere does not go through the center of rotation. In contrast, the observation of the demonstration experiment did not affect the inversion category, i.e., the students still would not choose a distractor that the sphere had been deflected to the left side in respect to the center of rotation if they had previously predicted that the trajectory is located on the right side of the disc. In this case, the choices were made by the same students.

The largest improvement between observe and explain in both reference frames, as a consequence of the instruction, is the frequency of the "confusion" category, i.e., after the instruction most students choose a distractor that relates a linear trajectory to the inertial frame of reference and a curved trajectory to the non-inertial frame of reference. Whereas the frequencies of distractors which belong to category III or IV are zero or close to zero after the instruction, again, as previously observed between predict and observe, the instruction did not affect the frequency of the inversion category and, again, the choices were made by the same students. This means that those students who previously chose a distractor that belongs to the inversion category, even after seeing the trajectory in an augmented photograph and in six videos, still tend to choose a distractor that shows that the trajectory in both coordinate systems is located on the right side of the disc.

\section{Student interviews}

The confidence ratings suggested that there are measurable, but negligible differences between distractor categories. To consolidate this finding and to identify students' conceptions we performed student interviews after completing the post-test. In these interviews we asked the students to comment on their answers of two particular questions Q1 and Q2 of the post-test. In question Q1 the students were asked to name the forces which are required to describe the trajectory of an airplane flying from the center of a rotating disc outwards in a uniform motion. In question Q2 the students were asked to predict whether or not water would slosh over the edge of a glass if the glass 
TABLE II. Distribution of answers of the two interview questions Q1 and Q2 (see Supplemental Material [77] for the questions and possible answers). The correct answer is marked with a dagger.

\begin{tabular}{lcc}
\hline \hline Distractor & Q1 [\%] & Q2 [\%] \\
\hline (a) & $28.6^{\dagger}$ & 0 \\
(b) & 0 & 4.8 \\
(c) & 42.8 & 14.3 \\
(d) & 28.6 & 4.8 \\
(e) & $\cdots$ & 23.8 \\
(f) & $\cdots$ & $52.8^{\dagger}$ \\
\hline \hline
\end{tabular}

moves along a curved trajectory in $K^{\prime}$ but uniformly along a straight line in $K$. The two questions and the possible answers are outlined in the Appendix. Language errors were corrected to improve readability. The interviews were conducted in German and, afterward, translated to English.

Table II shows the distribution of answers. In question Q1, the distractor (c) has the highest probability. This corresponds to the answer that only the Coriolis force is required to describe the trajectory of the airplane flying over a rotating disc. In the interviews, all students who chose this answer either justified their response by the thought that the airplane has no connection to the rotating disc or argued that in the absence of a centripetal force, no centrifugal one is required for the description of the trajectory. Here, we show two examples of medium performing students M1 and M2 and one example of a high performing student $\mathrm{H}$ :

Instructor: "Please comment on your answer of question Q1."

M1: "I ticked the third one because actually only the Coriolis force would have to work. I originally assumed that the Coriolis force is a counterforce of the centrifugal force. But since this is wrong and actually the centrifugal force is the counterforce of the centripetal force and since we have here, in my view, no centripetal force, there should be no centrifugal force here."

In the comments, student M1 describes the role of the centrifugal force as a counterforce to the centripetal force. This implies that the student does not apply the concept of the inertial centrifugal force and, instead, refers to the concept of the reactive centrifugal force, but mistakenly identifies an action-reaction pair of the centripetal and the centrifugal force.

And below is the answer of student M2, who comments to a wrong answer, in which the student assumes that only the Coriolis force is necessary to describe the trajectory of the airplane.

Instructor: "Please comment on your answer."

M2: "The plane is deflected to the left from the point of view of $K^{\prime}$ and as it flies and the air friction is neglected, it has no contact with the ground and therefore no centrifugal force has to act which somehow has to keep it on a circular path and therefore I think that you can neglect that. But if now a person would rest in the center of $K^{\prime}$, he would see that the aircraft is apparently being deflected to the left. The plane actually flies straight ahead, but the disc on which the observer stands turns to the right. And therefore, seen in the rotating system, only the Coriolis force acts which would deflect the aircraft."

Here, the student M2 argues that the missing contact of the airplane to the rotating frame of reference is responsible for the description via the Coriolis force.

For comparison, the following dialogue shows the correct reasoning of a student $\mathrm{H}$.

Instructor: "Please justify your answer of question Q1."

$\mathrm{H}$ : "To describe the trajectory in $K^{\prime}$, both the Coriolis and the centrifugal force are needed. This is the case because, first, we have a velocity of the airplane in the rotating frame of reference. That's why we need a Coriolis force. And since there is a distance $r^{\prime}$ to the center of rotation which is the origin of the coordinate system $K^{\prime}$, there must also be a centrifugal force."

In the arguments, student $\mathrm{H}$ directly refers to the nonzero velocity $v^{\prime}$ of the object in $K^{\prime}$ in the equation of the Coriolis force $F_{\text {Cor }}[\mathrm{Eq} .(\mathrm{A} 3)]$ and to the nonzero distance to the center of rotation $r^{\prime}$ which is a necessary component in the equation of the centrifugal force $F_{\text {Cen }}$ [Eq. (A4)].

Table III shows the distribution of reasoning to the interview question Q1. Here, the argument that the forces only act when the object is in contact with the rotating system, received the highest frequency of $42.9 \%$. In this argument, we did not discriminate between the students who chose the distractor that only the Coriolis force is required, and those students, who chose the distractor that no force is required for the description of the trajectory of the airplane in $K^{\prime}$, as long as the argument was the same. Apart from that, the reason that the absence of a centripetal force results in a situation, where the centrifugal force is not required for the description of the trajectory has the second highest frequency (19\%). Apart from these two reasons there was one student who argued that in a uniform motion there is no centrifugal force required for the description and one student guessed the answer.

In question Q2, the distractor (e) was chosen most frequently among the incorrect answers. This corresponds

TABLE III. Students' reasoning to interview question Q1 with corresponding frequency.

\begin{tabular}{lc}
\hline \hline Reasoning & Frequency [\%] \\
\hline $\begin{array}{l}\text { Forces act only when in contact } \\
\text { to the rotating system }\end{array}$ & 42.9 \\
No centripetal force results & 19.0 \\
$\quad$ in no centrifugal force & \\
Uniform motion means no centrifugal & 4.8 \\
$\quad$ force is required & \\
Guessed & 4.8 \\
Correct & 28.6 \\
\hline \hline
\end{tabular}


to the answer that the water is sloshed opposite to the direction of the sum vector of Coriolis and centrifugal force. This answer would be correct if there were real forces acting on the glass. This is the reason for the answer of student M1, who selected this answer:

M1: "The water spills out for sure because of the inertia of the water, so it's just a question of how it spills out and I've decided to tick the answer (e) because of the idea that the water goes straight ahead and thus the direction of motion is precisely directed opposite to these forces. Because it does not matter to the water, whether it is in the rotating system or not."

In this reasoning to question Q2, the student M1 seems convinced of the fact that Coriolis and centrifugal force cause an effect in the inertial frame $K$.

And this is the comment to the answer of the highachieving student $\mathrm{H}$ to question Q2:

$\mathrm{H}$ : "For the description of the trajectory, the Coriolis and centrifugal force are introduced and in $K$ the glass makes a straightforward uniform motion. But since both are only apparent forces, they are only of relevance for the trajectory description in $K^{\prime}$ and do not really affect the glass in the reference system $K$, the water does not spill over.

So, in this straight uniform motion, no force acts on the glass."

In this reasoning, the student $\mathrm{H}$ refers to the fictitious character of the Coriolis and centrifugal force and draws the correct solution by relating the uniform motion of the glass to the absence of forces in the stationary reference system $K$.

The reasoning of student M2 is similar to the one of $\mathrm{H}$, therefore it is not displayed here. Table IV shows the distribution of reasoning to the interview question Q2. In comparison to the distribution of answer alternatives in Table II, it is noticeable that all students, who chose a distractor responded with the same argument that inertial forces cause the same effect as real forces, even though they have chosen different answers. The reasoning includes comments about the inertia of the water and that the glass is located in $K^{\prime}$ so that the Coriolis and the centrifugal force act on the glass.

\section{Confidence levels affect visual focus}

We were interested whether confidence ratings following the predict and the observe tasks are correlated with measures of visual attention of the students. For this purpose, we divided the students in two groups: the first

TABLE IV. Students' reasoning to interview question Q2 with corresponding frequency.

\begin{tabular}{lc}
\hline \hline Reasoning & Frequency [\%] \\
\hline $\begin{array}{l}\text { Inertial forces cause the same effect } \\
\text { as real forces }\end{array}$ & 47.6 \\
Correct & 52.4 \\
\hline \hline
\end{tabular}

group rated their confidence in these items with confident or very confident, the students in the second group rated their confidence with "unconfident" or "very unconfident." Additionally, we analyzed the eye-tracking data in the following way. We designed a pattern of squarelike regions, so called areas of interest (AOIs), on each question page. On each of these AOIs we extracted the total visit duration which refers to the total time a student looks at the AOI. In our case, the AOIs exhibited a size of $50 \times 50$ pixel that covers all relevant areas (including the figure of the rotating disc, the coordinate system and the distractors) except the question text (see Fig. 6). We also analyzed an AOI pattern which includes the question text, but there is no statistically significant difference in the total visit duration on the text between the confident and unconfident students during the predict $(p=0.14)$ and the observe $(p=0.73)$ task. An inclusion of an AOI on the text in the analysis does not affect the statistical significance of the results and the total visit duration on the text is not responsible for the visual attention difference between confident and unconfident students on relevant areas (see below). Furthermore, the analysis using a $50 \times 50$-pixel pattern was previously used by Klein et al. [50] and, in comparison to a specific choice of certain regions (such as one distractor or a figure) as AOIs, it allows an analysis of the data which is unbiased by the AOI choice of a researcher while maintaining a reasonable resolution of the covered area.

Then, we compared the total visit duration and the size of the regions of attention. Table $\mathrm{V}$ shows the visit duration and the number of AOIs $N_{\text {AOIs }}$ which received a high focus (i.e., a focus which is longer than the average focus of each student) of students who feel confident of their answer and those who are unconfident during the predict task. In this table and in Table VI we combined the confidence ratings from the inertial frame and the non-inertial frame (i.e., total number of confidence ratings $N=42$ in each table). Here,

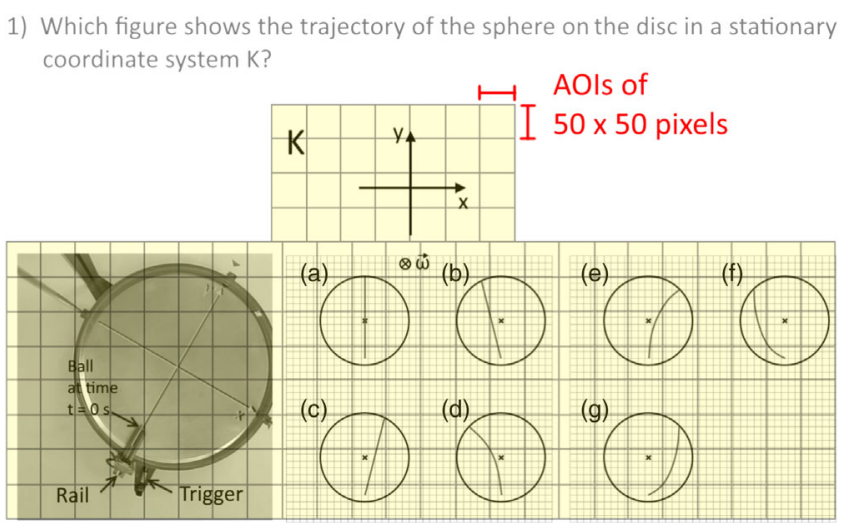

FIG. 6. Locations of square-shaped AOIs $(50 \times 50$ pixels, yellow areas) during the predict and observe task. Please note that the background of the answer alternatives is illustrated with a small squared pattern (not the AOIs). 
TABLE V. Maximum, average, and total visit duration on AOIs in seconds during the predict task as well as the number of AOIs $\left(N_{\mathrm{AOIs}}\right)$ that exhibit a visit duration larger than the average one. Additionally, the table contains the standard errors for each measured quantity.

\begin{tabular}{lccrl}
\hline \hline & $\begin{array}{c}\text { Confident } \\
(N=28)\end{array}$ & $\begin{array}{c}\text { Unconfident } \\
(N=14)\end{array}$ & $d$ & \multicolumn{1}{c}{$p$} \\
\hline Max [s] & $3.38 \pm 0.51$ & $7.33 \pm 1.12$ & 1.21 & 0.005 \\
Average [s] & $0.47 \pm 0.05$ & $0.76 \pm 0.08$ & 1.11 & 0.005 \\
$N_{\text {AOIs }}$ & $14.29 \pm 1.13$ & $13.86 \pm 1.45$ & -0.07 & 0.82 \\
Total [s] & $25.75 \pm 4.2$ & $44.56 \pm 6.7$ & 0.82 & 0.03 \\
\hline \hline
\end{tabular}

$N_{\text {AOIs }}$ is a measure of the size of the area of focus, i.e., it indicates the spatial spread of attention.

The analysis demonstrates that there is a statistically significant very large effect size in the maximum visit duration and a significant large effect in the average and total visit duration between confident and unconfident students during the predict questions (classification of effect sizes is according to Cohen [78]).

Table VI shows the visit duration and the number of AOIs that received high attention. It is noticeable that the differences in the maximum, average, and total visit duration of confident and unconfident students show the same trends as during the predict questions. Although the relative difference between confident and unconfident students is very similar in the predict and the observe part, the absolute value of the difference between confident and unconfident students is reduced in comparison to the predict questions. And, since the spread in the data is similar in Table V and Table VI, the differences between confident and unconfident students are not significant during the observe questions. Furthermore, the results indicate that there is no significant difference in $N_{\text {AOIs }}$ between confident and unconfident students which means that the studied area from where information is processed is similar between these two student groups. However, in comparison to the predict questions, $N_{\text {AOIs }}$ in the observe questions decreased more strongly

TABLE VI. Maximum, average, and total visit duration on AOIs during the observe task in seconds as well as the number of AOIs $\left(N_{\mathrm{AOIs}}\right)$ that exhibit a visit duration longer than the average one. The table also contains the standard errors for each measured quantity.

\begin{tabular}{lcccc}
\hline \hline & $\begin{array}{c}\text { Confident } \\
(N=29)\end{array}$ & $\begin{array}{c}\text { Unconfident } \\
(N=13)\end{array}$ & $d$ & $p$ \\
\hline Max [s] & $2.74 \pm 0.48$ & $5.68 \pm 1.9$ & 0.68 & 0.16 \\
Average [s] & $0.45 \pm 0.06$ & $0.65 \pm 0.13$ & 0.53 & 0.20 \\
$N_{\text {AOIs }}$ & $8.03 \pm 0.73$ & $11.77 \pm 1.69$ & 0.79 & 0.06 \\
Total [s] & $14.00 \pm 3.12$ & $31.60 \pm 9.94$ & 0.73 & 0.11 \\
\hline \hline
\end{tabular}

for confident students than for unconfident students which suggests that unconfident students spend more time extracting the same information, even though they have seen the same questions already before. Here, we cannot determine whether the differences in $N_{\mathrm{AOIs}}$ between predict and observe are significant or not since the groups are only partially paired.

On the one hand, in 22 out of 28 cases, where the answer was rated confident either in the inertial or in the noninertial frame in the predict part, the students also chose a confident rating to the corresponding question in the observe part, i.e., in 6 cases the students switched from a confident to an unconfident rating in the corresponding question in the observe part. On the other hand, in 7 out of 14 cases, where the answer was rated unconfident either in the inertial or in the non-inertial frame in the predict part, the students also chose an unconfident rating to the corresponding question in the observe part, i.e., in 7 cases the students switched from an unconfident to an confident rating in the corresponding question in the observe part.

\section{DISCUSSION}

In this work we demonstrated how students understand concepts of rotating frames of reference and how they apply their knowledge to understand a standard lecture experiment in which they are supposed to report the trajectory of a sphere rolling over a rotating disc in a rotating and in a stationary coordinate system.

The presented study reveals a number of preconceptions in the field of non-inertial frames of reference which lead to several promising suggestions for future instructions of the topic.

The distractor category I (confusion of $K$ and $K^{\prime}$ ) which includes those distractors that show a linear trajectory of the sphere in the non-inertial frame of reference or a curved trajectory of the sphere in the inertial frame, was the category with the highest frequency in the predict as well as in the observe items. The frequency of these distractors was significantly reduced after the instruction which consisted of a fundamental theoretical review of rotating frames of references, augmented photographs and six augmented videos. However, the results highlight the high difficulty of this topic for first-year physics students. This yields, for instance, the surprising observation that the distractors which contain the inversion error are neither corrected during the observation of the experiment nor during the instruction. This indicates that some errors require special attention which potentially could be realized via the implementation of cues [79] or via highlighting and discussing common errors of students in advance. For this reason, it is likely that a briefer instruction could fail to transfer the link between mathematical equations of the Coriolis and centrifugal force and their application to the trajectory of the sphere in a rotating and stationary frame of reference. 


\section{A. Influence of a lecture experiment on learning about the outcome of the demonstration (1st research question)}

Regarding the 1st research question, the item difficulty of the POE tasks is consistent with the conceptual and perceptual complexity of the topic of non-inertial frames of references. Only one out of five physics students was able to report the observation of the trajectory of a sphere rolling over the disc correctly in a single choice question. This is a lower rate than previous reports of POE interventions [33,34], which cover topics of mechanics, electricity, and magnetism, but do not include demonstration experiments regarding the motion of objects in non-inertial frames of reference. When the students passively observed the experiment, the frequency of correct statements about the outcome of the experiment was significantly increased (9\% higher) in comparison to students who did not see the experiment at all [34]. When students predicted the outcome of the experiment prior to the demonstration, the students' statement about the outcome after the demonstration was about 7\% higher than without previous prediction and $16 \%$ higher in comparison to students who did not see the experiment. This importance of the prediction of the experiment prior to the demonstration was later on confirmed by Miller et al. [33]. In contrast, in our work, there was no statistically significant increase in the frequency of correct responses about the outcome of the experiment between predict and observe. However, the detailed analysis of chosen distractors of the single choice questions during POE in combination with the identification of different distractor categories points towards specific benefits of the demonstration regarding the outcome of the experiment. The frequency of all distractor types show decreasing trends between predict and observe with the exception of the distractors that contain an inversion error. We found that distractors which incorrectly had no initial tangential velocity, were not chosen after observing the experiment. In this way, this type of analysis reveals specific benefits of the experiment demonstration, but the impact of the demonstration is significantly lower than previous reports.

\section{B. Preconceptions related to Coriolis and centrifugal force (2nd research question)}

The student interviews reveal prevailing preconceptions among first-semester physics students in the field of noninertial frames of reference. Nearly half of the participants $(42.9 \%)$ believe that the centrifugal force and sometimes also the Coriolis force are only necessary to describe the trajectory of an object in a rotating coordinate system if there is a coupling of the object to the rotating system. This conception is likely to be attributed to common instructional connections of the inertial centrifugal force and the reactive centrifugal force which occurs as a consequence to a centripetal force, such as the statement that "the centrifugal force disappears if the centripetal force would" [80], "This centrifugal force may be regarded as the reaction to the centripetal force" [81] or, in general, in experiments of rotating objects $[82,83]$. In the light of these results, we suggest to verbally discriminate between these two types of centrifugal forces. Apart from that, about one out of four physics students $(23.8 \%)$ do not include the fictitious character of inertial forces in their arguments and rather argue that they have the same effect on objects as real forces.

\section{Eye tracking reveals confidence (3rd research question)}

The eye-tracking analysis reveals a direct link between confidence ratings and visit duration on AOIs during the items of the predict phase. Students which are confident of their answer spent significantly less time on the AOIs than unconfident students. Despite this fact, unconfident students distribute their attention on a similar-sized area. This observation is an interesting extension to previous results and interpretations of long visit durations. For instance, Palinko et al. report that high visual attention on relevant areas is related to a high mental effort [84]. As a consequence, the visit duration has also been used as a measure for (intrinsic or extraneous) cognitive load within the framework of the cognitive load theory [85]. During the observe phase, there is no difference in the average or total visit duration between confident and unconfident students. The disappearance of the aforementioned relation between visit duration and confidence ratings in the observe part might be attributed to the fact that the students have seen the exact same questions already during the predict phase and have naturally less time on task since the content of the page is already partially familiar to the students. This interpretation is supported by an overall decrease of visit durations.

Furthermore, we observe that students with low confidence levels and high visit durations in the predict items distribute their focus on a similar-sized area as confident students. This seems to indicate that unconfident students tried longer to extract the same amount of information as confident students. In the theoretical framework of Rau [76], the author points towards necessary prerequisites for learning using multiple visual representations. To identify and extract relevant information from a visual representation such as a graph, photograph, or schematic, students need visual representational understanding which refers to the conceptual knowledge of how a visual representation depicts information. In order to relate the information from two different visual representations, as is required in several parts of this study, the students need connectional understanding of two or more representations. This knowledge refers to the ability to identify relevant similarities between the representation and to know about conventions for interpreting and combining the information from multiple representations [76]. Embedding our results in this framework, it seems that unconfident students seem to try to 
develop visual and/or connectional understanding of the representations displayed in the predict items.

\section{CONCLUSION}

In this study we tested the conceptual learning of physics students during a POE task on rotating frames of reference. The students had significant difficulties in predicting and observing the correct trajectory of a sphere (total score of approx. 20\%) rolling over a rotating disc in a stationary and a rotating coordinate system $K$ and $K^{\prime}$. Primarily, the low score can be attributed to the choice of a distractor which is consistent with a confusion of the effects of inertial forces in $K$ and $K^{\prime}$. Additionally, we found that some distractor choices even withstood the instruction. Students who initially predicted that the sphere is deflected to the opposite side on the disc (in respect to the actual trajectory), did not change their distractor choice during the observe and explain part (inversion category). This emphasizes the need for additional instructional support in this topic, for instance, via cues which highlight essential information.

Furthermore, the results indicate that after the instruction nearly half of the students answered that a centrifugal force will only be necessary if there is a coupling between the object and the rotating system. In comparison, the conception that an object shows a reaction to inertial forces in the same way as they do to real objects only persists in one-quarter of the students.

Within the POE task, the eye-tracking analysis in combination with confidence ratings showed that unconfident students spent significantly more time extracting information than confident students. This finding demonstrates the cognitive activation particularly of unconfident students during the predict phase. In contrast to previous reports, we found that passive observations of experiments, in fact, stimulate conceptual learning in a detailed distractor analysis which is not reflected in an increase of total scores. At this point we cannot judge the importance of this nonobvious learning behavior and additional research is necessary. Nevertheless, the results assist us to understand conceptual learning during POE tasks.

\section{ACKNOWLEDGMENTS}

This work is funded by the Federal Ministry of Education and Research (BMBF; project: VorleXung; support code: 16DHL1001). The authors are responsible for the content of this contribution.

\section{APPENDIX: THEORETICAL AND EXPERIMENTAL DETAILS \\ 1. Theoretical background on rotating frames of reference}

When an observer examines motion of an object moving uniformly in a stationary frame of reference (SFR) from a rotating frame of reference (RFR), the trajectory appears to be curved in comparison to a trajectory which a stationary observer (SO) would report. For instance, if an object moves uniformly in a SFR, it would display a curved trajectory for a rotating observer (RO). The theoretical description of the trajectory in a RFR requires the introduction of the centrifugal and the Coriolis force. They are "fictitious forces" which means that Newton's third law of motion $($ action $=$ reaction $)$ does not hold for them. In other words, both forces are not the result of an interaction between two bodies, but the consequence of the motion of the RFR. They are also called "inertial forces," which emphasizes the fact that the forces are caused by the inertia of the moving object. Typical examples include the motion of clouds observed from the earth or a thrown ball observed from a person sitting in a rotating merry go round [14].

The velocity $\vec{v}^{\prime}$ of an object in a RFR which rotates with a constant angular velocity $\vec{\omega}$ is given by the sum of the velocity $\vec{v}$ of the object with position $\vec{r}$ in the SFR and the negative tangential velocity $-\vec{\omega} \times \vec{r}$ in the RFR:

$$
\vec{v}^{\prime}=\vec{v}-\vec{\omega} \times \vec{r} .
$$

The derivative $d \vec{v}^{\prime} / d t$ leads to the acceleration of the object in the RFR [14]:

$$
\vec{a}^{\prime}=\vec{a}+\vec{\omega} \times(\vec{r} \times \vec{\omega})+2\left(\vec{v}^{\prime} \times \vec{\omega}\right) .
$$

This equation shows the necessity of introducing additional terms apart from the acceleration $\vec{a}$ in the SFR for the mathematical description of the determination of $\vec{a}^{\prime}$. The second term in Eq. (A2) corresponds to the inertial centrifugal acceleration and points radially outwards from the axis of rotation. The third term is called the Coriolis acceleration which is perpendicular to the velocity vector $\vec{v}^{\prime}$ in the plane of motion.

From Eq. (A2) the terms for the Coriolis force follow:

$$
\vec{F}_{\text {Cor }}=2 m\left(\vec{v}^{\prime} \times \vec{\omega}\right),
$$

and the equation for the centrifugal force

$$
\vec{F}_{\text {Cen }}=m \vec{\omega} \times(\vec{r} \times \vec{\omega}) .
$$

In both equations $m$ denotes the mass of the object.

\section{The change in speed $\left|\vec{v}^{\prime}\right|$ of an airplane flying over a rotating disc}

The problem of an airplane flying over an rotating disc can be described in polar (cylindrical) coordinates. For simplicity, the airplane flies with a speed $v_{0}$ in $x$ direction (see Fig. 7). In general the angle $\alpha^{\prime}$ of the airplane in the non-inertial frame is given by $\alpha^{\prime}=\alpha-\varphi$, where $\varphi$ is the rotational angle between the inertial and the non-inertial frame and $\alpha$ is the angle of the airplane in the inertial frame. Since the airplane moves in $x$ direction, $\alpha=0^{\circ}$ and thus 


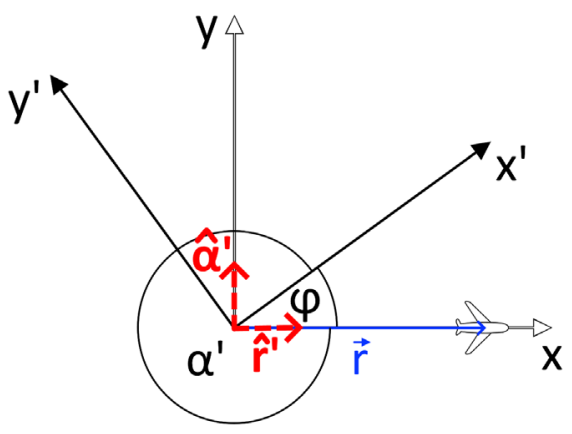

FIG. 7. Schematic of an airplane flying over a rotating disc. The inertial frame $K$ (represented by the axes $x$ and $y$ ) and the non-inertial frame $K^{\prime}$ (represented by the axes $x^{\prime}$ and $y^{\prime}$ ) share the same origin. For simplicity, the $z$ axis is not shown.

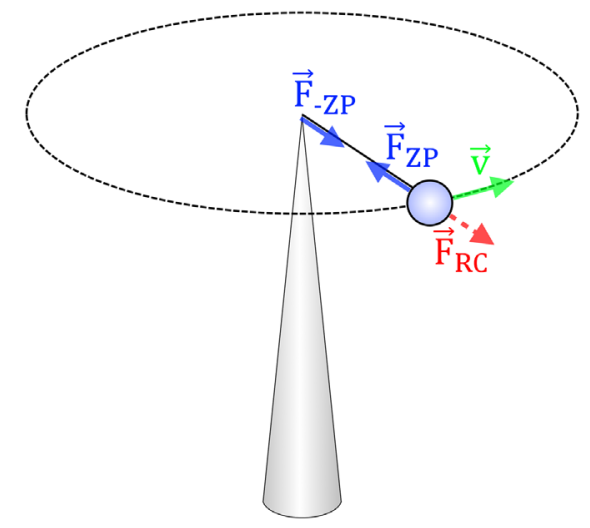

FIG. 8. Example of the reactive centrifugal force (RCF) $\vec{F}_{R C}$ of a ball attached to a post performing a circular motion. In the inertial frame only the centripetal force $\vec{F}_{Z P}$ and the reaction $\vec{F}_{-Z P}$ to the centripetal force occur. In the non-inertial frame, the RCF $\vec{F}_{R C}$ occurs additionally to the forces in the inertial frame. $\alpha^{\prime}=-\varphi\left(\right.$ or $\left.\alpha^{\prime}=360^{\circ}-\varphi\right)$. Let us assume that the noninertial frame rotates with the same rotational velocity $\dot{\alpha}^{\prime}$ as the disc and the center of the non-inertial frame is the same as the center of the inertial frame. Then, the position of the airplane in the inertial frame is given by

$$
\vec{r}=r \cos \alpha \hat{x}+r \sin \alpha \hat{y}=r \cdot \hat{r},
$$

with the unit vectors $\hat{x}$ and $\hat{y}$ in Cartesian coordinates in the inertial frame and the radial unit vector $\hat{r}=\cos \alpha \hat{x}+\sin \alpha \hat{y}$ in polar coordinates. Accordingly, in the non-inertial frame the position is determined by

$$
\vec{r}^{\prime}=r \cos \alpha^{\prime} \hat{x}^{\prime}+r \sin \alpha^{\prime} \hat{y}^{\prime}=r \cdot \hat{r}^{\prime},
$$

with $\hat{r}^{\prime}=\cos \alpha^{\prime} \hat{x}^{\prime}+\sin \alpha^{\prime} \hat{y}^{\prime}$ and $r=r^{\prime}=v_{0} t$. The derivative of the position $r^{\prime}$ in the non-inertial frame yields the velocity of the airplane in the non-inertial frame:

$$
\dot{\vec{r}}^{\prime}=\dot{r}^{\prime} \hat{r}^{\prime}+r^{\prime} \dot{\alpha}^{\prime} \hat{\alpha}^{\prime}=v_{0} \hat{r}^{\prime}+v_{0} t \dot{\alpha}^{\prime} \hat{\alpha}^{\prime} .
$$

Here, the angular unit vector in polar coordinates is given by

$$
\hat{\alpha}^{\prime}=-\sin \alpha^{\prime} \hat{x}+\cos \alpha^{\prime} \hat{y} .
$$

Consequently, the absolute value of the velocity in the non-inertial frame of reference is

$$
\left|\vec{v}^{\prime}\right|=\left|\dot{\vec{r}}^{\prime}\right|=\sqrt{v_{0}^{2}+v_{0}^{2} t^{2} \dot{\alpha}^{\prime 2}}
$$

From Eq. (A9) it follows that the absolute value $\left|\dot{\vec{r}}^{\prime}\right|$ of the airplane velocity in the non-inertial frame increases with time $t$. Therefore, an observer in the non-inertial frame notices an increase in the absolute value of the velocity, even though the airplane flies in a uniform motion over the disc.

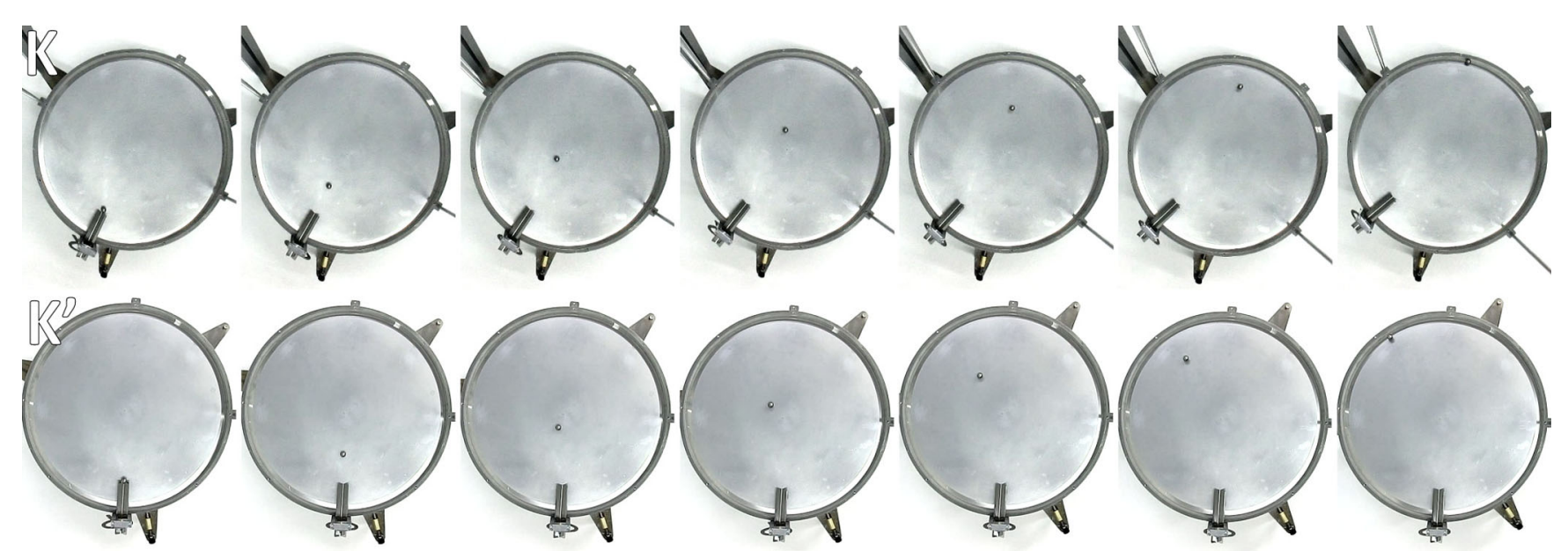

FIG. 9. Snapshots of videos taken in the inertial frame of reference $K$ (top row) and the non-inertial frame of reference $K^{\prime}$ (bottom row). There is a time difference of $0.17 \mathrm{~s}$ between two subsequent snapshots. 


\section{Example of the reactive centrifugal force}

In this example, we consider a ball that is attached to a post while it performs a circular motion (Fig. 8). Here, the centripetal force $\vec{F}_{Z P}$ acts on the ball and it forms a actionreaction pair with the force $\vec{F}_{-Z P}$ which acts on the post. The RCF $\vec{F}_{R C}$ also acts on the ball but in the same direction as $\vec{F}_{-Z P} . \vec{F}_{R C}$ does not form a action-reaction pair with another force.

The RFC needs to be introduced in case the motion is described in a rotating frame of reference (non-inertial frame) which has the origin at the position of the post and rotates with the same angular velocity as the ball. In this case, the ball rests in the non-inertial frame which means that apart from the centripetal force which acts on the ball another force is required which balances the centripetal force. For this purpose, the RCF is introduced.

\section{Snapshots of videos from different perspectives}

The video which represents the inertial frame of reference, was recorded using the camera which is attached to the outer frame. The top row in Fig. 9 represents seven snapshots from this video. The video which shows the motion of the sphere in the non-inertial frame of reference, was recorded using the camera which is rotating with the disc. Seven snapshots from this camera are shown in the bottom row of Fig. 9.
[1] J. R. Holton and G. J. Hakim, An Introduction to Dynamic Meteorology (Academic Press, New York, 2012).

[2] A. Persson, The Coriolis effect: Four centuries of conflict between common sense and mathematics, Part I: A history to 1885, Proceedings of the International Commission on History of Meteorology, Beijing (2005), Vol. 2, pp. 1-24.

[3] J. T. Kirby, F. Shi, B. Tehranirad, J. C. Harris, and S. T. Grilli, Dispersive tsunami waves in the ocean: Model equations and sensitivity to dispersion and Coriolis effects, Ocean Model. 62, 39 (2013).

[4] B. Dintrans and M. Rieutord, Oscillations of a rotating star: A non-perturbative theory, Astron. Astrophys. 354, 86 (2000).

[5] K. Y. Bliokh, Y. Gorodetski, V. Kleiner, and E. Hasman, Coriolis Effect in Optics: Unified Geometric Phase and Spin-Hall Effect, Phys. Rev. Lett. 101, 030404 (2008).

[6] P. Walker and G. Dracoulis, Energy traps in atomic nuclei, Nature 399, 35 (1999).

[7] Example open online material demonstrating the Coriolis effect: Youtube National Geographic, https://www.youtube .com/watch?v=mPsLanVS1Q8 (December 2018).

[8] Example of an explanation of the Coriolis Effect: The Coriolis effect describes the pattern of deflection taken by objects not firmly connected to the ground as they travel long distances around and above the Earth. National Geographic: https://www.nationalgeographic.org/encyclopedia/ coriolis-effect/ (August 2019).

[9] The effect of the Coriolis force is an apparent deflection of the path of an object that moves within a rotating coordinate system. https://www.britannica.com/science/ Coriolis-force (August 2019).

[10] The Coriolis effect (also known as the Coriolis force) refers to the apparent deflection of objects (such as airplanes, wind, missiles, and ocean currents) moving in a straight path relative to the Earth's surface. https://www.thoughtco .com/what-is-the-coriolis-effect-1435315 (August 2019).
[11] Anything traveling long distances, like air currents, ocean currents pushed by air, and airplanes, will all be deflected because of the Coriolis Effect! https://scijinks.gov/coriolis/ (August 2019).

[12] Example open online material demonstrating the Coriolis effect: Nova, What the Physics?!, https://www.youtube .com/watch?v=6L5UD240mCQ (August 2019).

[13] https://www.youtube.com/watch?v=dt_XJp77-mk.

[14] W. Demtröder, Mechanics and Thermodynamics (Springer, New York, 2017).

[15] W. Nolting, Theoretical physics 1: Classical Mechanics (Springer, New York, 2016).

[16] D. Kleppner and R. Kolenkow, An Introduction to Mechanics (Cambridge University Press, Cambridge, England, 2014).

[17] T. W. Kibble and F. H. Berkshire, Classical Mechanics (World Scientific Publishing Company, Singapore, 2004).

[18] M. J. Benacquista and J. D. Romano, Classical Mechanics (Springer, New York, 2018).

[19] C. Kittel, W. D. Knight, M. A. Ruderman, A. C. Helmholz, and B. J. Moyer, Mechanics (Berkeley Physics Course, Vol. 1). Vectors, 26, 3. (McGraw-Hill, New York, NY, 1962).

[20] D. Halliday and R. Resnick, Physics, Combined Edition, Parts 1 and 2 (John Wiley and Sons, New York, 1978), 3rd ed.

[21] T. Dorfmüller, L. Bergmann, and C. Schaefer, Mechanik, Relativität, Wärme, Vol. 1 (Walter de Gruyter, Berlin-New York, 1998).

[22] H. Goldstein, C. Poole, and J. Safko, Classical Mechanics (Addison-Wesley, New York, 2002).

[23] D. Morin, Introduction to Classical Mechanics: With Problems and Solutions (Cambridge University Press, Cambridge, England, 2008).

[24] P. A. Tipler and G. Mosca, Physics for Scientists and Engineers (Macmillan Higher Education, London, 2008).

[25] R. P. Feynman, R. B. Leighton, and M. Sands, The Feynman Lectures on Physics: Mainly Mechanics, Radiation and Heat I (Addison-Wesley, Reading, MA, 1963). 
[26] R. Müller, Klassische Mechanik: Vom Weitsprung zum Marsflug (Walter de Gruyter, Berlin, 2010).

[27] D. Meschede, Gerthsen Physik (Springer-Verlag, Berlin, 2015).

[28] A. Persson, How do we understand the Coriolis force?, Bull. Am. Meteorol. Soc. 79, 1373 (1998).

[29] J. Roche, Introducing motion in a circle, Phys. Educ. 36, 399 (2001).

[30] Y. Kobayashi, Remarks on viewing situation in a rotating frame, Eur. J. Phys. 29, 599 (2008).

[31] H. C. Corben and P. Stehle, Classical Mechanics (Courier Corporation, New York, 2013).

[32] H. M. Stommel and D. W. Moore, An Introduction to the Coriolis Force (Columbia University Press, New York, 1989).

[33] K. Miller, N. Lasry, K. Chu, and E. Mazur, Role of physics lecture demonstrations in conceptual learning, Phys. Rev. ST Phys. Educ. Res. 9, 020113 (2013).

[34] C. Crouch, A. P. Fagen, J. P. Callan, and E. Mazur, Classroom demonstrations: Learning tools or entertainment?, Am. J. Phys. 72, 835 (2004).

[35] R. White and R. Gunstone, Prediction-observationexplanation, Probing Understanding 1, 4 (1992).

[36] A. B. Champagne, L. E. Klopfer, and J. H. Anderson, Factors influencing the learning of classical mechanics, Am. J. Phys. 48, 1074 (1980).

[37] D. R. Sokoloff and R. K. Thornton, Using interactive lecture demonstrations to create an active learning environment, Phys. Teach. 35, 340 (1997).

[38] R. F. Gunstone, Children's science: A decade of developments in constructivist views of science teaching and learning, Aust. Sci. Teachers J. 36, 9 (1990).

[39] C. W. Liew and D. F. Treagust, A predict-observe-explain teaching sequence for learning about students understanding of heat and expansion of liquids, Aust. Sci. Teachers J. 41, 68 (1995).

[40] H. Schecker and R. Duit, Schlervorstellungen und Physikunterricht: Ein Lehrbuch fr Studium, Referendariat und Unterrichtspraxis, edited by H. Schecker, T. Wilhelm, M. Hopf, and R. Duit (Springer-Verlag, Berlin, Heidelberg, 2018).

[41] R. Duit, STCSE: Students and Teachers Conceptions and Science Education (Bibliography) (2009) Available from Leibniz-Institut für die Pädagogik der Naturwissenschaften http://archiv.ipn.uni-kiel.de/stcse/.

[42] R. Duit and D. F. Treagust, Conceptual change: A powerful framework for improving science teaching and learning, Int. J. Sci. Educ. 25, 671 (2003).

[43] J. Clement, Students preconceptions in introductory mechanics, Am. J. Phys. 50, 66 (1982).

[44] R. Gunstone and M. Watts, Force and motion, Children's Ideas Sci. 1, 85 (1985).

[45] M. A. Just and P. A. Carpenter, Eye fixations and cognitive processes, Cogn. Psychol. 8, 441 (1976).

[46] R. E. Mayer, Unique contributions of eye-tracking research to the study of learning with graphics, Learn. Instr. 20, 167 (2010).

[47] A. M. Madsen, A. M. Larson, L. C. Loschky, and N. S. Rebello, Differences in visual attention between those who correctly and incorrectly answer physics problems, Phys. Rev. ST Phys. Educ. Res. 8, 010122 (2012).

[48] M. J. Tsai, H. T. Hou, M. L. Lai, W. Y. Liu, and F. Y. Yang, Visual attention for solving multiple-choice science problem: An eye-tracking analysis., Comput. Educ. 58, 375 (2012).

[49] A. Susac, A. Bubic, E. Kazotti, M. Planinic, and M. Palmovic, Student understanding of graph slope and area under a graph: A comparison of physics and nonphysics students, Phys. Rev. Phys. Educ. Res. 14, 020109 (2018).

[50] P. Klein, S. Küchemann, S. Brückner, O. ZlatkinTroitschanskaia, and J. Kuhn, Student understanding of graph slope and area under a curve: A replication study comparing first-year physics and economics students, Phys. Rev. Phys. Educ. Res. 15, 020116 (2019).

[51] A. Madsen, A. Rouinfar, A. M. Larson, L. C. Loschky, and N.S. Rebello, N. S. Can short duration visual cues influence students reasoning and eye movements in physics problems?, Phys. Rev. ST Phys. Educ. Res. 9, 020104 (2013).

[52] P. Klein, J. Viiri, and J. Kuhn, Visual cues improve students understanding of divergence and curl: Evidence from eye movements during reading and problem solving, Phys. Rev. Phys. Educ. Res. 15, 010126 (2019).

[53] A. N. Susac, A. Bubic, J. Kaponja, M. Planinic, and M. Palmovic, Eye movements reveal students strategies in simple equation solving, Int. J. Sci. Math. Educ. 12, 555 (2014).

[54] K. A. Ericsson and A. C. Lehmann, Expert and exceptional performance: Evidence of maximal adaptation to task constraints, Annu. Rev. Psychol. 47, 273 (1996).

[55] A. Gegenfurtner, E. Lehtinen, and R. Slj, Expertise differences in the comprehension of visualizations: A meta-analysis of eye-tracking research in professional domains, Educ. Psychol. Rev. 23, 523 (2011).

[56] K. A. Ericsson and W. Kintsch, Long-term working memory, Psychol. Rev. 102, 211 (1995).

[57] H. Haider and P. A. Frensch, Eye movement during skill acquisition: More evidence for the information reduction hypothesis, J. Exper. Psychol. Learn. Memory Cogn. 25, 172 (1999).

[58] E. Ohno, A. Shimojo, and M. Iwata, Analysis of problem solving processes in physics based on eye-movement data, in The Conference of International Research Group on Physics Teaching (GIREP) European Physical SocietyPhysics Education Division (EPS PED) (University of Wrocław, Poland, 2015), p. 67.

[59] M. Kekule, Students different approaches to solving problems from kinematics in respect of good and poor performance, in Proceedings of the International Conference on Contemporary Issues in Education (ICCIE) (2015), p. 126-134.

[60] M. Kekule, Students approaches when dealing with kinematics graphs explored by eyetracking research method, in Proceedings of the Frontiers in Mathematics and Science Education Research Conference, FISER (2014), pp 108-117.

[61] D. Rosengrant, C. Thomson, and T. Mzoughi, Comparing experts and novices in solving electrical circuit problems with the help of eye-tracking, in Proceedings of the 2009 
Physics Education Research Conference, Ann Arbor, MI (AIP, New York, 2009), p. 24952.

[62] P. Klein, J. Viiri, S. Mozaffari, A. Dengel, and J. Kuhn, Instruction-based clinical eye-tracking study on the visual interpretation of divergence: How do students look at vector field plots?, Phys. Rev. Phys. Educ. Res. 14, 010116 (2018).

[63] T. van Gog, F. Paas, and J. J. G. van Merrienboer, Uncovering expertise-related differences in troubleshooting performance: Combining eye movement and concurrent verbal protocol data, Appl. Cogn. Psychol. 19, 205 (2005).

[64] A. C. Graesser, S. Lu, B. A. Olde, E. Cooper-Pye, and S. Whitten, Question asking and eye tracking during cognitive disequilibrium: Comprehending illustrated texts on devices when the devices break down, Mem. Cogn. 33, 1235 (2005).

[65] M. Kozhevnikov, M. A. Motes, and M. Hegarty, Spatial visualization in physics problem solving, Cogn. Sci. 31, 549 (2007).

[66] M. D. Sharma and J. Bewes, Self-monitoring: Confidence, academic achievement and gender differences in physics, J. Learn. Des. 4, 1 (2011).

[67] P. Klein, A. Müller, and J. Kuhn, Assessment of representational competence in kinematics, Phys. Rev. Phys. Educ. Res. 13, 010132 (2017).

[68] S. Kleitman and L. Stankov, Ecological and person oriented aspects of metacognitive processes in test taking, Appl. Cogn. Psychol. 15, 321 (2001).

[69] G. Gigerenzer, U. Hoffrage, and H. Kleinbölting, Probabilistic mental models: a Brunswikian theory of confidence, Psychol. Rev. 98, 506 (1991).

[70] B. Fischhoff, Debiasing, in Judgment Under Uncertainty: Heuristics and Biases Cambridge, edited by D. Kahneman, P. Slovic, and A. Tversky (Cambridge University Press, Cambridge, England, 1982), pp. 422-444.

[71] C. M. Allwood and H. Montgomery, Response selection strategies and realism of confidence judgments, Org. Behav. Human Dec. Proc. 39, 365 (1987).

[72] R. S. May and P. Lang, Realismus von subjektiven Wahrscheinlichkeiten: eine kognitionspsychologische Analyse inferentieller Prozesse beim Overconfidence-Phänomen, edited by $\mathrm{P}$. Lang Gmbh (Internationaler Verlag Der Wissenschaften, Bern, 1987), Vol. 205.

[73] M. Planinic, W. J. Boone, R. Krsnik, and M. L. Beilfuss, Exploring alternative conceptions from Newtonian dynamics and simple DC circuits: Links between item difficulty and item confidence, J. Res. Sci. Teach. 43, 150 (2006).
[74] Standards for the final high school diploma in physics can be found at https://gymnasium.bildung-rp.de/lehrplaeneepa-bildungsstandards-abitur.html.

[75] Standards for the final high school diploma in math can be found at https://www.kmk.org/fileadmin/Dateien/ veroeffentlichungen_beschluesse/2012/2012_10_18Bildungsstandards-Mathe-Abi.pdf.

[76] M. A. Rau, Conditions for the effectiveness of multiple visual representations in enhancing STEM learning, Educ. Psychol. Rev. 29, 717 (2017).

[77] See Supplemental Material at http://link.aps.org/ supplemental/10.1103/PhysRevPhysEducRes.16.010112 for pretest items, interview questions and instruction material.

[78] J. Cohen, Statistical Power Analysis for the Behavioral Sciences, 2nd ed. (Lawrence Erlbaum, Hillsdale, NJ, 1988), p. 56.

[79] A. Madsen, A. Rouinfar, A. M. Larson, L. C. Loschky, and N.S. Rebello, Can short duration visual cues influence students reasoning and eye movements in physics problems?, Phys. Rev. ST Phys. Educ. Res. 9, 020104 (2013).

[80] The statement was translated from the original phrase in German: "Da die Zentrifugalkraft nur ein anderer Ausdruck dafür ist, dass der Körper infolge seiner Trägheit sich der Richtungsänderung durch die Zentripetalkraft widersetzt, verschwindet sie gleichzeitig mit der letzteren.", from K. Lüders and G. Oppen, Mechanik, Akustik, Wärme, Vol. 1 (Walter de Gruyter, Berlin, 2008), p. 116.

[81] A. L. Reimann, Physics: Mechanics and Heat, Electricity, Magnetism, and Optics (Barnes \& Noble, New York, 1971), Vol. 1.

[82] Video demonstrating that the centrifugal force causes a deflection of a pendulum attached to a merry go round. The Science Asylum https://www.youtube.com/watch? v=zHpAifN_2Sw (January 2020).

[83] Video showing a simulation of a can attached to a string rotating in a circular motion. This video shows the error that the centrifugal force is the reaction to the centripetal force. MrRyanPitcher, https://www.youtube.com/watch? v=9s1IRJbL2Co (January 2020).

[84] O. Palinko, A. L. Kun, A. Shyrokov, and P. Heeman, Estimating cognitive load using remote eye tracking in a driving simulator, in Proceedings of the 2010 Symposium on Eye-Tracking Research \& Applications (ACM, New York, 2010), pp. 141-144.

[85] R. E. Mayer and R. Moreno, Nine ways to reduce cognitive load in multimedia learning, Educ. Psychol. 38, 43 (2003). 